\title{
Prenatal alcohol exposure did not affect impulsivity in rats that performed delay or probability discounting tasks
}

\author{
Stefano Pupe ${ }^{1}$, Ivani Brys ${ }^{1}$, Philip J. E. Asherson ${ }^{2}$ and Lisiane Bizarro ${ }^{1}$ \\ 1 - Universidade Federal do Rio Grande do Sul, Porto Alegre, RS, Brazil \\ 2 - King's College London, London, UK
}

\begin{abstract}
Individuals who fall under the spectrum of the Fetal Alcohol Syndrome have a higher prevalence of several cognitive disturbances, including a greater probability of being diagnosed with attention-deficit hyperactivity disorder (ADHD). Some of these effects, such as hyperactivity and attentional impairments, are already well established in the literature. The assessment of impulsive choice, however, has received little attention in human and animal studies. In the present study, we attempted to investigate the effects of prenatal ethanol exposure on two tasks related to impulsive choice that have never been studied in this condition: delay and probability discounting. Method: Rats prenatally exposed to ethanol (liquid diets with $0 \%, 10 \%$, or 35\% ethanol-derived calories [EDC] or laboratory chow) were trained to respond for food in either delay $(n=21)$ or probability $(n=48)$ discounting tasks performed in computer-controlled operant conditioning chambers. Results: Prenatal treatment failed to differentiate the rates at which the rats chose the larger reinforcer associated with delay - in a task in which 35\% EDC was not tested - or risk, although the results suggest that further tests are warranted. Keywords: delay discounting, probability discounting, Fetal Alcohol Syndrome, prenatal ethanol exposure, impulsivity
\end{abstract}

Received 24 March 2011; received in revised form 24 May 2011; accepted 24 May 2011. Available on line 15 June 2011

\section{Introduction}

In humans, ethanol is a known teratogen, causing abnormalities that range from severe Fetal Alcohol Syndrome ([FAS]; Jones \& Smith, 1973), a leading cause of mental retardation, to more subtle cognitive (Streissguth et al., 1986; Streissguth, Barr, \& Sampson, 1990; Olson, Sampson, Barr, Streissguth, \& Bookstein, 1992) and social impairments (Disney, Iacono, McGue, Tully, \& Legrand, 2008). Of these two findings are particularly relevant for the present study: the link between prenatal alcohol exposure and attentional/ response inhibition abnormalities when young (Brown et al., 1991; Streissguth et al., 1994) and drinking problems in adolescence and adulthood (Baer, Barr, Bookstein, Sampson, \& Streissguth, 1998; Baer, Sampson, Barr, Connor, \& Streissguth, 2003).

Stefano Pupe, Ivani Brys and Lisiane Bizarro, Laboratório de Psicologia, Neurociencias e Comportamento, Departamento de Psicologia do Desenvolvimento e da Personalidade, Instituto de Psicologia, Universidade Federal do Rio Grande do Sul / Unidade de Experimentação Animal, Hospital de Clínicas de Porto Alegre. Philip J. E. Asherson, MRC Social Genetic and Developmental Psychiatry, Institute of Psychiatry P080, King's College London, De Crespigny Park, London SE5 8AF, United Kingdom. Correspondence regarding this article should be directed to: Stéfano Pupe, Ramiro Barcelos 2600, Porto Alegre, Brazil. E-mail: stefanopj@gmail.com
Both of these factors appear to be related to impulsivity, particularly impulsive choice. Evidence suggests that the abuse of alcohol is correlated with the percentage of choices of immediate rewards in a delay discounting task (Field, Christiansen, Cole, \& Gouldie, 2007; Petry, 2001). Similarly, subjects diagnosed with attention-deficit hyperactive disorder (ADHD) tend to discount future rewards more steeply than controls (Barkley, Edwards, Laneri, Fletcher, \& Metevia, 2001; Kuntsi, Oosterlaan, \& Stevenson, 2001; Solanto et al., 2001). Prenatal alcohol exposure leads to some of the cognitive abnormalities seen in ADHD, and it is in fact considered a risk factor for this disorder (Mick, Biederman, Faraone, Sayer, \& Kleinman, 2002).

Prenatal alcohol exposure is also known to produce various disruptions of the dopaminergic pathways in the brain (Druse, Tajuddin, Kuo, \& Connerty, 1990; Shen, Hannigan, \& Kapatos, 1999; Choong \& Shen, 2004). These findings are relevant because dopaminergic pathways and regions related to them are important to tasks of impulsive choice (Cardinal \& Howes, 2005). An especially interesting structure is the nucleus accumbens, where abnormalities caused by prenatal alcohol were also observed (Blanchard et al., 1993; Chen, Maier, \& West, 1997). Lesions in this structure were shown to shift behavior in delay and probability discounting tasks toward delayed and uncertain rewards 
(Cardinal, Pennicott, Sugathapala, Robbins, \& Everitt, 2001; Cardinal \& Howes, 2005). Systemic injections of amphetamine appear to have the opposite effect in both tasks (Simon, Gilbert, Mayse, Bizon, \& Setlow, 2009; van Gaalen, van Koten, Schoffelmeer, \& Vanderschuren, 2006) although this effect appears to be mediated by the presence or absence of cues during the waiting period for a delayed reward, at least in delay discounting tasks (Cardinal, Robbins, \& Everitt, 2000). Some dopaminergic antagonists, such as raclopride and flupenthixol, also affect the delay discounting task by increasing impulsive choice (Wade, de Wit, \& Richards, 2000).

Considering these different types of converging evidence, an intriguing question is whether individuals exposed to ethanol in utero might exhibit differences in their patterns of choice mediated by time or probability, which reflect two dissociable aspects of impulsive choice (Myerson, Green, Hanson, Holt, \& Estle, 2003). The identification of different patterns of choice in delay and probability tasks is important because they underlie several theories regarding ADHD and impulsivity in general (Evenden, 1999). Individuals prenatally exposed to ethanol appear to have similar, yet distinguishable, cognitive and behavioral characteristics compared with subjects diagnosed with ADHD (Coles et al., 1997). The study of impulsive choice could provide further evidence regarding the possible differences between these two subgroups and contribute to a wider understanding of the effects of prenatal alcohol on impulsive choice. Although some evidence indicate that prenatal alcohol disrupts timing behavior (Wass, Simmons, Thomas, \& Riley, 2002; Simmons, Levy, Riley, Madra, \& Mattson, 2009) and motor impulsivity (Olmstead, Martin, Brien, \& Reynolds, 2009), to our knowledge, no direct tests of delay or probability discounting have been reported in the literature.

To address that, we performed these tests in an animal model. Using groups of rats exposed to two different doses of prenatal alcohol with the use of liquid diets, together with pair-fed controls, we sought to replicate a level of exposure that is below that of full-blown FAS. The objective was to verify whether prenatal ethanol exposure interferes with impulsive choice in delay and probability discounting tasks.

\section{Methods}

\section{Subjects}

The subjects were the offspring of 80 female (primiparous) and 40 male Wistar rats from Fundação Estadual de Produção e Pesquisa em Saúde (Rio Grande do Sul, Brazil). The females were weighed and placed into weight-matched groups. The dams were randomly assigned to one of four maternal treatments: (i) A35 (i.e., liquid diet with 35\% ethanol-derived calories), (ii) A10 (i.e., liquid diet with $10 \%$ ethanol-derived calories), (iii) Control (i.e., liquid diet without ethanol), and (iv) Chow (i.e., free access to laboratory chow and water). The liquid diets were a commercially available formulation (Bio-Serv, Frenchtown, NJ, USA), with a nutritional composition based on the work of Lieber and DeCarli (1989). The rats had 1 week of habituation to the liquid diets before the period of mating began, during which the diet administration was interrupted. The diets were returned to each rat when its pregnancy was confirmed by vaginal smear and remained available until the day they gave birth, after which all groups were fed normally with ad libitum laboratory chow and water. During pregnancy, the experimental procedure was adapted from Clausing et al. (1996). The A35 group had access to an ad libitum liquid diet each day, and the volume consumed by each dam on a given gestational day was the limit provided to its weight-matched counterparts in the A10 and Control groups on the same gestational day. Because the diets were much more palatable for the A10 and Control groups than for the A35 group, the rats from the former groups always consumed everything that was available to them. This procedure ensured that each triplet (one dam in each experimental group) consumed the same volume of diet in the same gestational day, thus keeping the caloric intake constant, with only the amount of ethanol-derived calories varying between groups. National and institutional guidelines for animal welfare were followed, and all procedures were approved by the Ethics Committee of the Hospital de Clínicas de Porto Alegre.

Separate groups of siblings were used for the two tasks (i.e., each individual rat performed only one of the tasks). Dams exposed to the higher dose of ethanol had an increased number of stillborn pups (18vs. 1 in the A10 group and 0 in the other groups). On postnatal day 21 (PND21), A35 litters had 50\% fewer live pups compared with the other groups. For this reason, the A35 group was used in only one task (probability discounting). Only male rats were used for all tasks.

\section{Apparatus}

Five-hole nose poke chambers with soundattenuating cubicles (Med Associates, St. Albans, VT, USA) were used. Each chamber had a receptacle attached to a pellet dispenser and a house light on one side and five smaller apertures on the opposite wall. For the purposes of this task, only the left-most (Aperture 1) and right-most (Aperture 2) apertures were used. Every aperture was equipped with LED lights and infrared sensors. The chambers were controlled by computer software (MED-PC IV, Med Associates).

\section{Training}

The animals were housed in pairs and gradually restricted to $85 \%$ of their free-feeding weight. One week before testing, sucrose pellets were sprinkled in their home cages for habituation. Training began under 
a simple fixed-ratio schedule, in which rats had to nose poke one of the apertures to obtain a $45 \mathrm{mg}$ sucrose pellet (Bio-Serv) delivered in the receptacle. The rats were trained to a criterion of 50 correct responses (defined as nose pokes inside a lit aperture) within a 30 min session, first for Aperture 1 and then for Aperture 2. This stage lasted for approximately 5 days and was the same for both tasks.

\section{Delay discounting}

This task was performed on PND60 with 21 rats (seven from each group; A35 rats were not used). The delay discounting procedures followed the protocol used by Cardinal et al. (2000) using solely the "houselight" paradigm of their study. The task consisted of associating one aperture with a smaller, immediate reward (one pellet) and another aperture with a bigger, delayed reward (four pellets). The delay in this latter option progressively increased during the five blocks of the task $(0,10,20,40$, and $60 \mathrm{~s})$, each of which consisted of two forced choice trials and 10 free choice trials. In the forced choice trials, each aperture (immediate and delayed) was individually lit once. During the free choice trials, both apertures were lit simultaneously.

Each trial was initiated with an intertrial period (100 s), in which all lights were kept off. Afterward, the house light and light in the feeder receptacle were lit. The rat had to perform a nose poke in this receptacle, after which its light went off, and Aperture 1 and/or (depending on whether it was a free or forced choice trial) Aperture 2 lit up. If the rat chose the aperture associated with the delayed reward, then a delay period followed, in which the house light was kept on, with its duration determined by the current block. After this period, four pellets were dispensed, the house and feeder lights were lit, and after the rat collected the reward, the apparatus returned to the intertrial state. If the rat instead chose the aperture associated with the immediate reward, then it was immediately rewarded with one pellet. The rat had $10 \mathrm{~s}$ to begin the trial, 10 $\mathrm{s}$ to select any option, and $10 \mathrm{~s}$ to collect the reward. An omission was recorded if the rat failed to do so within this time period. Each experimental session had 60 trials (12 for each block), each lasting $100 \mathrm{~s}$, and the duration of every daily session was $100 \mathrm{~min}$. The association between Aperture 1 or Aperture 2 and the delayed option was randomly assigned for each rat and remained stable throughout the sessions.

Only the data from the free choice trials were analyzed. The criterion for achieving baseline was the analysis of the last seven sessions for each rat using two-way analysis of variance (ANOVA), with Delay and Day as factors. In this analysis, the rats had to show both a significant effect of delay $(p<.05)$ and a nonsignificant effect of day $(p>.05)$ to be considered for further analysis. The mean number of choices of the delayed aperture in each block, the number of omissions, and the latency to initiate a trial were measured for each group using the average data from these last seven sessions.

\section{Probability discounting}

For this test, which began on PND120, 48 rats were used (A35, $n=8$; A10, $n=16$; Control, $n=12$; Chow, $n=12)$. The procedures, adapted from Nasrallah, Yang, and Bernstein (2009), were somewhat similar to the delay discounting task, with the distinction that, after the training period, one aperture corresponded to a bigger (four pellets) uncertain reward $(75 \%, 50 \%$, etc., chance of being presented) and the other to a smaller (one pellet) reward (100\% chance of being presented). In both cases, the reward, if forthcoming, was presented immediately after the choice was made. The objective was to compare the pattern of responses of the animals in each group with the percentage of choices made in the uncertain option. Most of the procedures were identical to the delay discounting task, with the exception of the differences explained below.

The probability of reward in the uncertain option was changed only from one day to the next and was fixed for every session. Each daily 45 min session was separated into two blocks. The first block was composed of forced choice trials, in which a single option was available to the animal at a time. In the second block, the animal was free to choose between the certain and uncertain rewards. During both blocks, if the animal chose the uncertain option and no reward was dispensed, then all lights were turned off, and the chamber returned to its intertrial state. Each block was composed of 24 trials, and each trial had a fixed duration of $55 \mathrm{~s}$, regardless of choice or omissions.

No baseline performance was required for this test, which was run in two separate phases. The first lasted 1 week, and the probabilities associated with the larger reward were progressively lowered each day in the following order: $100 \%, 75 \%, 50 \%, 25 \%$, and $16 \%$. However, to ensure that the pattern of choices observed was not attributable to a carry-over effect from multiple sessions, 4 more weeks of testing were performed. During each week, the first day had a 100\% probability of delivering a bigger reward, and the other 4 days had probabilities of $16.66 \%, 25 \%, 33.33 \%$, and $66.66 \%$ for each week, which remained stable for the whole week. The order in which the rats performed in each probability was constant for all rats but followed a predetermined sequence of $25 \%, 66.66 \%, 33.33 \%$, and $16,66 \%$ from the first to fourth week, respectively.

Only the data from the free choice trials were analyzed. For the first phase of this task, a repeatedmeasures ANOVA was used to compare the performance during the entire week, with Session and Group as factors. For the second phase, the same procedure was 
used, but only the last day of each week was considered for the analysis to ensure that it reflected more clearly the pattern of choice associated with that probability. The dependent measures were the percentage of choice of the larger reward, total omissions, and the average latency to initiate a trial.

Importantly, no "true" randomness was used in this task, as it would possibly lead to vastly different experiences for each animal, denying the possibility of actually controlling the probability of reward. For that reason, this experiment used a design in which, although the probability of reward in each individual trial was controlled pseudorandomly by a function in the program (i.e., it was, in practical terms, unpredictable), the overall probability of a set of trials was fixed. In a set of 24 trials, for example, if the probability of reward was set to $50 \%$ and the animal chose the uncertain option in every trial, then it would be rewarded exactly 12 times.

\section{Results}

\section{Delay discounting}

At the beginning of the task, the weight of the animals was not significantly different between groups, with mean weights varying between 304 and $310 \mathrm{~g}$ for the three groups. After 45 sessions, all of the animals were able to achieve a stable baseline, with the exception of one subject from the Chow group, which was excluded from the analysis because its pattern of choices did not change in any block. The assumption of homogeneity of variance was met for all factors and variables, with the exception of omissions. A Mauchly's test of sphericity indicated that sphericity was broken for all variables tested (percentage of choice, omissions, and latency; $p<.001)$. Therefore, all subsequent results reflect Greenhouse-Geisser corrected values. Figure 1 shows the mean percentage of choice of the delayed option for each group in the different blocks.

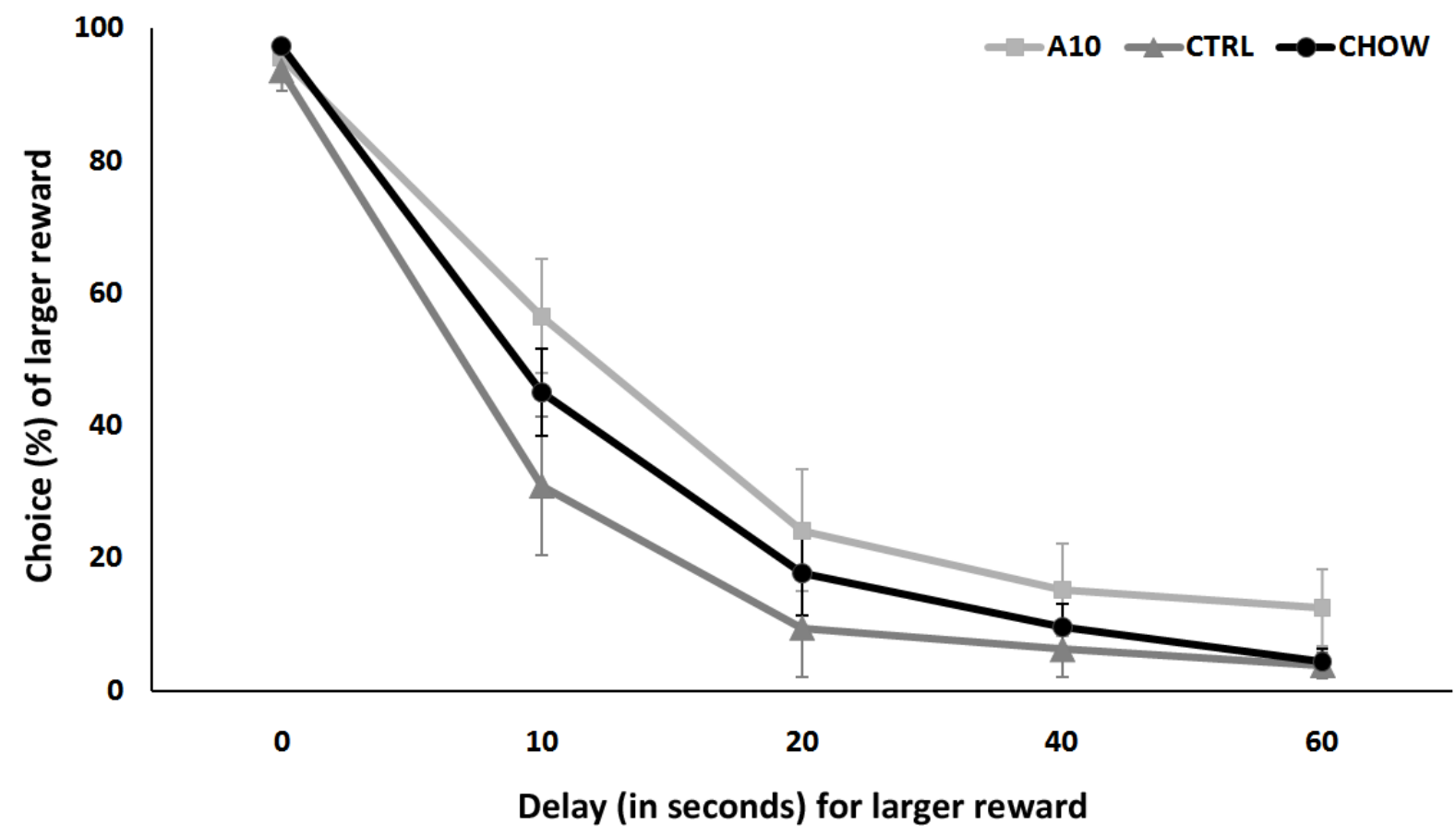

Figure 1. Mean percentage ( $\pm S E M$ ) of choice of the larger, delayed reward with respect to the delay (in seconds) associated with the choice. No significant difference between groups was found.

Table 1. Mean ( $\pm S E M)$ number of omissions and latency to initiate trials during each block of 10 trials with different delays (D1 to D5) associated with the larger reward.

\begin{tabular}{lllllll}
\hline & & D1 $(0 \mathrm{~s})$ & D2 (10s) & D3 (20s) & D4 (40s) & D5 (60s) \\
\hline Omissions & A10 & $0.2(0.2)$ & $1.3(0.6)$ & $1.6(0.7)$ & $1.6(0.7)$ & $1.6(0.7)$ \\
& CTRL & 0 & $0.2(0.1)$ & $0.3(0.1)$ & $0.4(0.2)$ & $0.3(0.2)$ \\
& CHOW & 0 & $0.2(0.1)$ & $0.2(0.1)$ & $0.3(0.1)$ & $0.2(0.1)$ \\
\hline \multirow{2}{*}{ Latency (s) to initiate trial } & A10 & $1.7(0.3)$ & $2.0(0.2)$ & $2.3(0.3)$ & $2.5(0.3)$ & $2.4(0.3)$ \\
& CTRL & $1.5(0.1)$ & $1.8(0.1)$ & $2.1(0.1)$ & $1.9(0.2)$ & $2.1(0.2)$ \\
& CHOW & $1.5(0.3)$ & $1.9(0.3)$ & $1.9(0.3)$ & $2.1(0.4)$ & $1.9(0.3)$ \\
\hline
\end{tabular}


An ANOVA showed no significant differences between the three groups in the choice of delayed options in each block $(F[17,2]=1.52 ; p=.24)$. The same was true for the number of omissions $(F[17,2]=$ $2.54 ; p=.10)$ and latency to initiate trials $(F[17,2]=$ $0.44 ; p=.64$; Table 1 ).

\section{Probability discounting}

The animals in the A35 group had a significantly lower weight compared with all of the other groups, which was expected according to the developmental effects of prenatal alcohol. The mean weights $( \pm S E M)$ were, respectively, 390 (5.5), 440 (10.5), 439 (13.5), and $456(10) \mathrm{g}$ for the A35, A10, Control, and Chow groups. The homogeneity of variance assumption was met only for the second phase of testing, for all variables tested. In the first phase, it was not met for some of the variables (particularly omissions) and test conditions. Again, all of the reported data are Greenhouse-Geisser corrected value, as a Mauchly's test of sphericity indicated that the assumption of sphericity was broken $(p<.001)$. The data from the first week of testing revealed that the Session factor had a significant impact on performance $(F[75.8,1.7]$ $=29.13 ; p<.001$ ), which was expected given the different probabilities associated with each session. However, the effect of Group was not statistically significant $(F[44,3]=2.14 ; p=.10)$, and no interaction between these factors was found $(F[75.8,5.1]=1.18$; $p=.32$; Figure 3 ). With regard to omissions and the latency to initiate trials, neither Day nor Group were significant factors $(p<.05$; Table 2$)$.

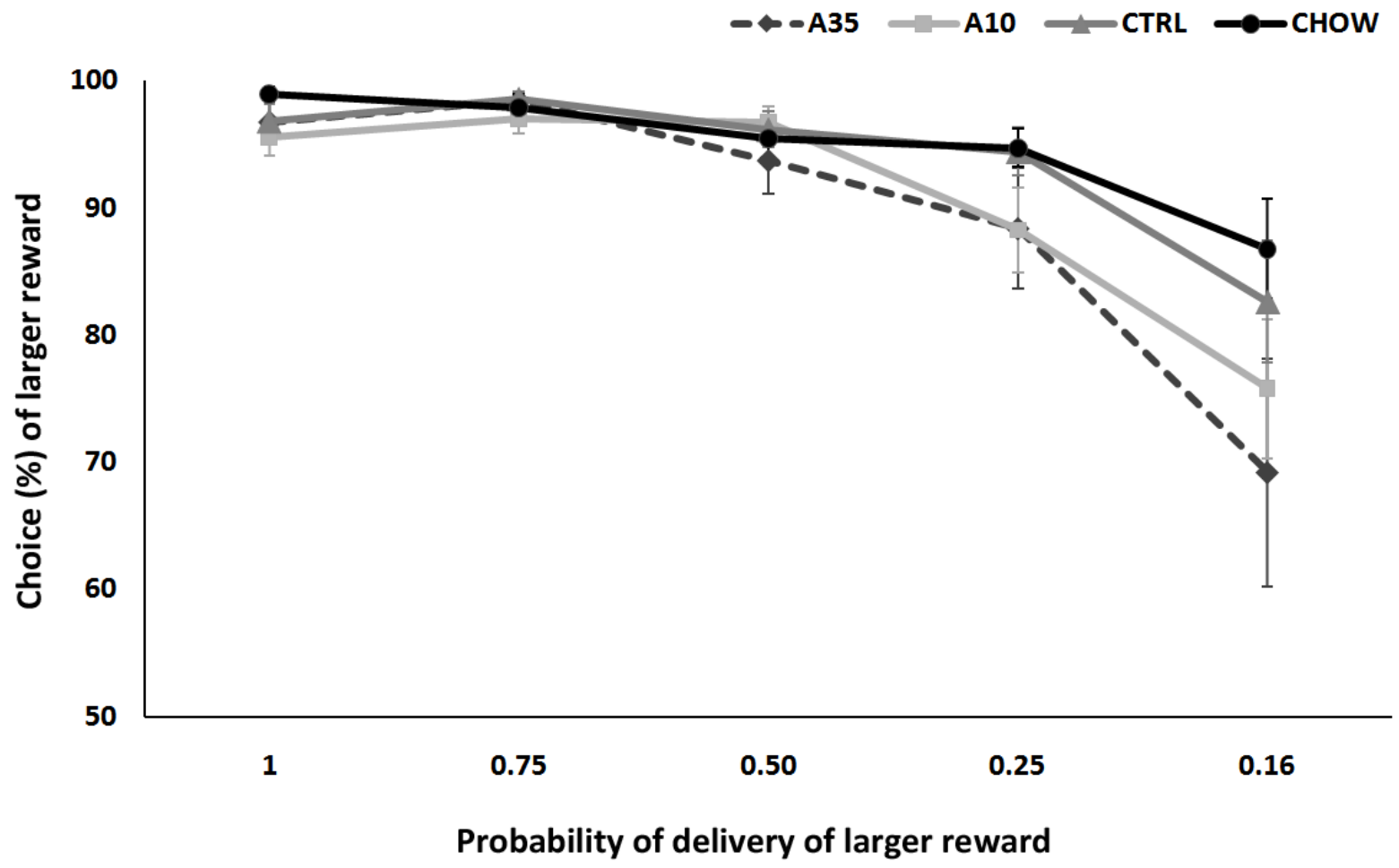

Figure 2. Mean percentage $( \pm S E M)$ of choice of larger, uncertain reward in each session and its corresponding probability of being delivered. These results were not significantly different $(p=.10)$.

Table 2. Mean $( \pm S E M)$ number of omissions and latency to initiate trials in the first phase of testing with different probabilities (P1-P5) of release of the larger reward.

\begin{tabular}{lllllll}
\hline & & P1 (100\%) & P2 (75\%) & P3 (50\%) & P4 (25\%) & P5 (16\%) \\
\hline Omissions & A35 & $2.1(1.0)$ & $0.8(0.6)$ & 0 & $0.1(0.1)$ & $0.1(0.1)$ \\
& A10 & $1.7(1.1)$ & $1.6(0.8)$ & $0.1(0.1)$ & $0.1(0.1)$ & $0.1(0.1)$ \\
& CTRL & $0.4(0.3)$ & $0.4(0.2)$ & 0 & $0.3(0.2)$ & 0 \\
& CHOW & $0.6(0.5)$ & $0.4(0.2)$ & $0.1(0.1)$ & 0 & 0 \\
\hline Latency (s) to initiate trial & A35 & $3.7(0.5)$ & $3.1(0.5)$ & $2.3(0.3)$ & $1.8(0.1)$ & $2.0(0.1)$ \\
& A10 & $3.1(0.5)$ & $2.6(0.3)$ & $1.9(0.1)$ & $1.9(0.2)$ & $2.1(0.2)$ \\
& CTRL & $2.4(0.4)$ & $2.0(0.3)$ & $1.9(0.1)$ & $1.9(0.1)$ & $2.0(0.1)$ \\
& CHOW & $2.9(0.4)$ & $2.5(0.2)$ & $2.0(1.5)$ & $1.9(0.8)$ & $1.9(0.1)$ \\
\hline
\end{tabular}




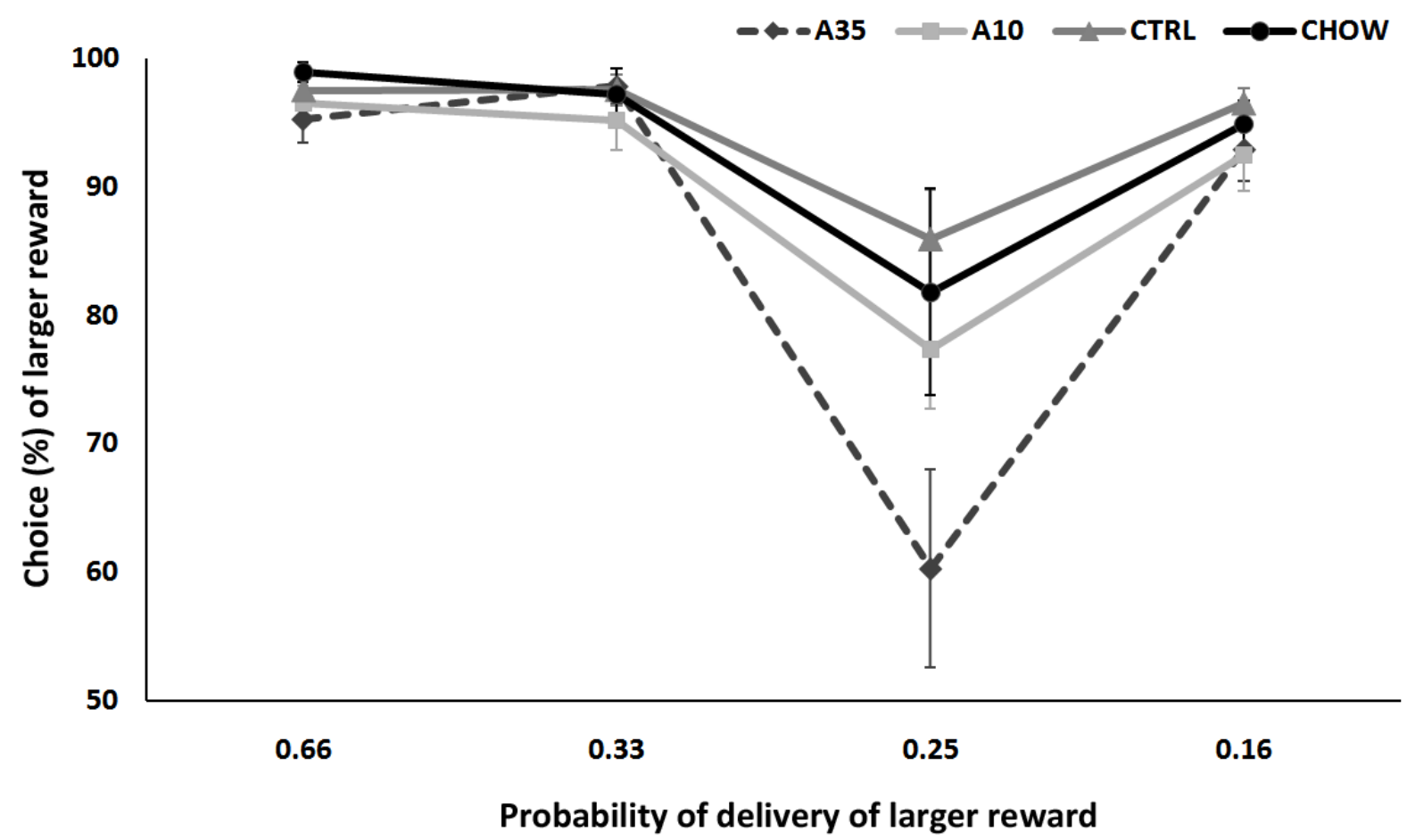

Figure 3. Mean $( \pm S E M)$ percentage of choice of the uncertain reward according to its corresponding probability of being delivered on the last day of each week in the second phase of testing. Again, these results were not significant $(p=.07)$.

Table 3. Mean $( \pm S E M)$ number of omissions and latency to initiate trials in the second phase of testing associated with different probabilities (P1-P4) of release of the larger reward.

\begin{tabular}{llllll}
\hline & & P1 $(66 \%)$ & P2 $(33 \%)$ & P3 $(25 \%)$ & P4 (16\%) \\
\hline Omissions & A35 & $2.0(0.8)$ & $0.8(0.4)$ & $1.1(0.5)$ & $0.6(0.3)$ \\
& A10 & $1.6(0.6)$ & $0.8(0.2)$ & $1.1(0.3)$ & $1.0(0.4)$ \\
& CTRL & $2.0(0.8)$ & $0.9(0.3)$ & $2.0(0.4)$ & $1.1(0.4)$ \\
& CHOW & $2.1(0.8)$ & $0.4(0.2)$ & $0.6(0.3)$ & $0.7(0.3)$ \\
\hline Latency (s) to initiate trial & A35 & $2.3(0.3)$ & $1.7(0.1)$ & $1.9(0.1)$ & $2.1(0.2)$ \\
& A10 & $2.1(0.2)$ & $2.0(0.1)$ & $2.3(0.2)$ & $2.4(0.2)$ \\
& CTRL & $2.2(0.2)$ & $2.1(0.1)$ & $2.2(0.1)$ & $2.3(0.3)$ \\
& CHOW & $2.5(0.2)$ & $1.8(0.1)$ & $2.0(0.1)$ & $2.2(0.1)$ \\
\hline
\end{tabular}

In the second phase of testing, the probabilities were kept stable for 1 week, and only the data from the last day of each week were analyzed. Again, the Session factor was significant $(F[67.4,1.5]=35.58 ; p<.001)$, but neither an effect of Group $(F[44,3]=2.46 ; p=.07)$ nor an interaction $(F[67.4,4.5]=2.04 ; p=.08)$ was found (Figure 3 ). The omissions and latencies to initiate trials were not affected by Session or Group ( $p>.5$; Table 3$)$.

\section{Discussion}

The present results are evidence that, in contrast to several kinds of cognitive damage mentioned in the Introduction, prenatal exposure to ethanol does not appear to affect the pattern of impulsive choice in adulthood. This was true for delay discounting, although only a single dose of ethanol was tested in this task, and probability discounting. In the latter test, some interesting patterns of behavior emerged, which are discussed in consideration of the available literature.

The first striking characteristic is that, when tested with progressively diminishing probabilities in a single week, the results were mostly consistent with what is expected of a pattern of choice facing diminishing odds. However, when the animals faced the same odds in the second phase of testing, their choices were inconsistent with their previous behavior, in which the choice of larger rewards paradoxically increased when they were less likely. The reversal happened at exactly a point at which the better option was to switch to the certain, smaller rewards because the odds for the uncertain rewards did not compensate for their magnitude (16.66\% chance of receiving a four-fold larger reward). 
Based on the available evidence, this reversal of preference over time was not expected. Studies that have tested rats during adolescence and adulthood found very similar, albeit not identical, results at both stages of development (Nasrallah et al., 2009; Adriani et al., 2005). One difference is that the previous studies used the same protocol-one week of testing with diminishing odds - in both cases times, while we changed the experimental procedure by keeping the probabilities stable over 1 week. This factor likely played a role in the present results, suggesting that choices that involve probabilistic rewards are highly susceptible to slight differences in testing, particularly ones that involve the residual effect of previous contingencies.

With regard to the disadvantageous pattern of choices performed by all groups when the probabilities were small, the evidence is conflicting. The groups that used a different protocol, one that encompassed many different probabilities in a single session through the use of blocks of trials, found that the rats adapted particularly well to small, disadvantageous probabilities, adjusting their percentage of choice of uncertain rewards accordingly (Cardinal \& Howes, 2005; St. Onge \& Floresco, 2008). The same happened in a study that used procedures that were very similar to the ones used in the present study (Nasrallah et al., 2009). A possible explanation lies in the differences between strains. The aforementioned studies used Long Evans or Lister Hooded rats. Two studies that used Wistar rats and procedures analogous to ours also found an inconsistent pattern of choice, with rats increasing their preference for larger, uncertain rewards when they became economically disadvantageous (Adriani et al., 2005; Adriani \& Laviola, 2006). Indeed, strain differences were found to be significant in delay discounting tasks (Oberlin \& Grahame, 2009; Wilhelm $\&$ Mitchell, 2009). The only study that compared strains in the probability discounting task used high and low alcohol-drinking strains (Wilhelm, \& Mitchell, 2008), indicating that the question of whether Wistar rats are particularly less risk averse remains unanswered.

Notably, using the same strain that we did and using a protocol similar to ours, Adriani et al. (2005) were able to show significant differences between their experimental groups, finding that rats exposed to methylphenidate showed a more economically advantageous pattern of choices. In our case, the significance values obtained, particularly in the first week of training, tentatively suggest that the A35 group avoided more risks compared with controls. However, this conclusion is undermined by the fact that the results failed to achieve a conventional level of significance, and because of the aforementioned inconsistent results in both phases of testing.

Nevertheless, these results indicate that further studies of impulsive choice in individuals prenatally exposed to ethanol are warranted. In the delay discounting task, a wider range of doses of alcohol need to be investigated. For the probability discounting test, the use of different protocols, species, or strains might provide interesting results. The issue of determining what types of longlasting cognitive impairment can be caused by alcohol during pregnancy is an important one, particularly with low-to-moderate doses. The testing of impulsive choice in both humans and animals is a widely underrepresented subset of this field.

\section{Acknowledgements}

SP and IB were funded by grants from the Conselho Nacional de Desenvolvimento Científico e Tecnológico. This study was also funded by the Wellcome Trust (grant no. $079314 / \mathrm{Z} / 06 / \mathrm{Z}$ ). The authors would like to thank Marta Cioato, Fabíola Schons Meyer, Carolina Mello, and Eduardo Amaro da Silveira for their outstanding quality of animal care and Ian P. Stolerman for his support of this work.

\section{References}

Adriani, W., Leo, D., Greco, D., Rea, M., di Porzio, U., Laviola, G., \& Perrone-Capano, C. (2005). Methylphenidate administration to adolescent rats determines plastic changes on reward-related behavior and striatal gene expression. Neuropsychopharmacology, 31, 1946-1956.

Adriani, W., \& Laviola, G. (2006). Delay aversion but preference for large and rare rewards in two choice tasks: implications for the measurement of self-control parameters. BMC Neuroscience, 7(1), 52.

Baer, J.S., Barr, H.M., Bookstein, F.L., Sampson, P.D., \& Streissguth, A.P. (1998). Prenatal alcohol exposure and family history of alcoholism in the etiology of adolescent alcohol problems. Journal of Studies on Alcohol, 59, 533-543.

Baer, J.S., Sampson, P.D., Barr, H.M., Connor, P.D., \& Streissguth, A.P. (2003). A 21-year longitudinal analysis of the effects of prenatal alcohol exposure on young adult drinking. Archives of General Psychiatry, 60, 377-385.

Barkley, R.A., Edwards, G., Laneri, M., Fletcher, K., \& Metevia, L. (2001). Executive functioning, temporal discounting, and sense of time in adolescents with attention deficit hyperactivity disorder (ADHD) and oppositional defiant disorder (ODD). Journal of Abnormal Child Psychology, 29, 541-556.

Blanchard, B.A., Steindorf, S., Wang, S., LeFevre, R., Mankes, R.F., \& Glick, S.D. (1993). Prenatal ethanol exposure alters ethanolinduced dopamine release in nucleus accumbens and striatum in male and female rats. Alcoholism: Clinical and Experimental Research, 17, 974-981.

Brown, R.T., Coles, C.D., Smith, I.E., Platzman, K.A., Silverstein, J., Erickson, S., \& Falek, A. (1991). Effects of prenatal alcohol exposure at school age: I. Attention and behavior. Neurotoxicology and Teratology, 13, 369-376.

Cardinal, R.N., Robbins, T.W., \& Everitt, B.J. (2000). The effects of d-amphetamine, chlordiazepoxide, a-flupenthixol and behavioural manipulations on choice of signalled and unsignalled delayed reinforcement in rats. Psychopharmacology, 152, 362-375.

Cardinal, R.N., \& Howes, N.J. (2005). Effects of lesions of the nucleus accumbens core on choice between small certain rewards and large uncertain rewards in rats. BMC Neuroscience, 6, 37.

Cardinal, R.N., Pennicott, D.R., Sugathapala, C.L., Robbins, T.W., \& Everitt, B.J. (2001). Impulsive choice induced in rats by lesions of the nucleus accumbens core. Science, 292, 2499-2501.

Chen, W.J., Maier, S.E., \& West, J.R. (1997). Prenatal alcohol treatment attenuated postnatal cocaine-induced elevation of dopamine concentration in nucleus accumbens: a preliminary study. Neurotoxicology and Teratology, 19(1), 39-46.

Choong, K., \& Shen, R. (2004). Prenatal ethanol exposure alters the 
postnatal development of the spontaneous electrical activity of dopamine neurons in the ventral tegmental area. Neuroscience, 126, 1083-1091.

Clausing, P., Ali, S.F., Taylor, L.D., Newport, G.D., Rybak, S., \& Paule, M.G. (1996). Central and peripheral neurochemical alterations and immune effects of prenatal alcohol exposure in rats. International Journal of Developmental Neuroscience, 14, 461-469.

Coles, C.D., Platzman, K.A., Raskind-Hood, C.L., Brown, R.T., Falek, A., \& Smith, I.E. (1997). A comparison of children affected by prenatal alcohol exposure and attention deficit, hyperactivity disorder. Alcoholism: Clinical and Experimental Research, 21(1), 150-161.

Disney, E.R., Iacono, W., McGue, M., Tully, E., \& Legrand, L. (2008). Strengthening the case: prenatal alcohol exposure is associated with increased risk for conduct disorder. Pediatrics, 122, e1225-e1230.

Druse, M.J., Tajuddin, N., Kuo, A., \& Connerty, M. (1990). Effects of in utero ethanol exposure on the developing dopaminergic system in rats. Journal of Neuroscience Research, 27, 233-240.

Evenden, J.L. (1999). Varieties of impulsivity. Psychopharmacology, 146, 348-361.

Field, M., Christiansen, P., Cole, J., \& Gouldie, A. (2007). Delay discounting and the alcohol stroop in heavy drinking adolescents. Addiction, 102, 579-586.

Jones, K.L., \& Smith, D.W. (1973). Recognition of the fetal alcohol syndrome in early infancy. Lancet, 2, 999-1001.

Lieber, C.S., \& DeCarli, L.M. (1989). Liquid diet technique of ethanol administration: 1989 update. Alcohol and Alcoholism, 24, 197-211.

Kuntsi, J., Oosterlaan, J., \& Stevenson, J. (2001). Psychological mechanisms in hyperactivity: I Response inhibition deficit, working memory impairment, delay aversion, or something else? Journal of Child Psychology and Psychiatry, 42, 199-210.

Mick, E., Biederman, J., Faraone, S.V., Sayer, J., \& Kleinman, S. (2002). Case-control study of attention-deficit hyperactivity disorder and maternal smoking, alcohol use, and drug use during pregnancy. Journal of the American Academy of Child and Adolescent Psychiatry, 41, 378-385.

Myerson, J., Green, L., Hanson, J.S., Holt, D.D., \& Estle, S.J. (2003). Discounting delayed and probabilistic rewards: processes and traits. Journal of Economic Psychology, 24, 619-635.

Nasrallah, N.A., Yang, T.W.H., \& Bernstein, I.L. (2009). Longterm risk preference and suboptimal decision making following adolescent alcohol use. Proceedings of the National Academy of Sciences of the United States of America, 106, 17600-17604.

Oberlin, B.G., \& Grahame, N.J. (2009). High-alcohol preferring mice are more impulsive than low-alcohol preferring mice as measured in the delay discounting task. Alcoholism: Clinical and Experimental research, 33, 1294-1303.

Olmstead, M.C., Martin, A., Brien, J.F., \& Reynolds, J.N. (2009). Chronic prenatal ethanol exposure increases disinhibition and perseverative responding in the adult guinea pig. Behavioural Pharmacology, 20, 554-557.

Olson, H.C., Sampson, P.D., Barr, H., Streissguth, A.P., \& Bookstein,
F.L. (1992). Prenatal exposure to alcohol and school problems in late childhood: a longitudinal prospective study. Development and Psychopathology, 4, 341-359.

Petry, N.M. (2001). Delay discounting of money and alcohol in actively using alcoholics, currently abstinent alcoholics, and controls. Psychopharmacology, 154, 243-250.

Shen, R., Hannigan, J.H., \& Kapatos, G. (1999). Prenatal ethanol reduces the activity of adult midbrain dopamine neurons. Alcoholism: Clinical and Experimental Research, 23, 1801-1807.

Simmons, R.W., Levy, S.S., Riley, E.P., Madra, N.M., \& Mattson, S.N. (2009). Central and peripheral timing variability in children with heavy prenatal alcohol exposure. Alcoholism: Clinical and Experimental Research, 33, 400-407.

Simon, N.W., Gilbert, R.J., Mayse, J.D., Bizon, J.L., \& Setlow, B. (2009). Balancing risk and reward: a rat model of risky decisionmaking. Neuropsychopharmacology, 34,2208-2217.

Solanto, M., Abikoff, H., Sonuga-Barke, E., Schachar, R., Logan, G., Wigal, T., ...Turkel, E. (2001). The ecological validity of delay aversion and response inhibition as measures of impulsivity in $\mathrm{AD} /$ HD: a supplement to the NIMH multimodal treatment study of AD/ HD. Journal of Abnormal Child Psychology, 29, 215-228.

St. Onge, J.R., \& Floresco, S.B. (2008). Dopaminergic modulation of risk-based decision making. Neuropsychopharmacology, 34, 681-697.

Streissguth, A.P., Barr, H.M., Sampson, P.D., Parrish-Johnson, J.C., Kirchner, G.L., \& Martin, D.C. (1986). Attention, distraction and reaction time at age 7 years and prenatal alcohol exposure. Neurobehavioral Toxicology and Teratology, 8, 717-725.

Streissguth, A.P., Barr, H.M., \& Sampson, P.D. (1990). Moderate prenatal alcohol exposure: effects on child IQ and learning problems at age 7 1/2 years. Alcoholism: Clinical and Experimental Research, 14, 662-669.

Streissguth, A.P., Sampson, P.D., Olson, H.C., Bookstein, F.L., Barr, H.M., Scott, M., Feldman, J., \& Mirsky, A.F. (1994). Maternal drinking during pregnancy: attention and short-term memory in 14-year-old offspring: a longitudinal prospective study. Alcoholism: Clinical and Experimental Research, 18, 202-218.

van Gaalen, M.M., van Koten, R., Schoffelmeer, A.N., \& Vanderschuren, L.J. (2006). Critical involvement of dopaminergic neurotransmission in impulsive decision making. Biological Psychiatry, 60(1), 66-73.

Wade, T.R., de Wit, H., \& Richards, J.B. (2000). Effects of dopaminergic drugs on delayed reward as a measure of impulsive behavior in rats. Psychopharmacology, 150, 90-101.

Wass, T.S., Simmons, R.W., Thomas, J.D., \& Riley, E.P. (2002). Timing accuracy and variability in children with prenatal exposure to alcohol. Alcoholism: Clinical and Experimental Research, 26, 1887-1896.

Wilhelm, C.J., \& Mitchell, S.H. (2008). Rats bred for high alcohol drinking are more sensitive to delayed and probabilistic outcomes. Genes, Brain, and Behavior, 7,705-713.

Wilhelm, C.J., \& Mitchell, S.H. (2009). Strain differences in delay discounting using inbred rats. Genes, Brain, and Behavior, 8, 426-434. 